\title{
The efficacy and safety of azithromycin in treatment for childhood asthma: a systematic review and meta-analysis
}

\author{
Xiangcheng Pan ${ }^{1}$, Yan $\mathrm{Liu}^{1}$, Jiefeng $\mathrm{Luo}^{1}$, Siyu $\mathrm{Li}^{2}$, Sha Diao ${ }^{2}$, Hailong $\mathrm{Li}^{2}$, Liang Huang${ }^{2}$, \\ Zhijun $\mathrm{Jia}^{2}$, Lina Chen ${ }^{2}$, Hanmin $\mathrm{Liu}^{2}$, and Lingli Zhang ${ }^{1}$ \\ ${ }^{1}$ Sichuan University \\ ${ }^{2}$ Sichuan University West China Second University Hospital
}

May 29, 2021

\begin{abstract}
Background: Evidence suggests controversial results based on the antibacterial and anti-inflammatory effects of azithromycin (AZI) in the treatment of childhood asthma. This study was to further evaluate the efficacy and safety of AZI in childhood asthma. Methods: We searched PubMed, Embase (via Ovid), Cochrane Library, China National Knowledge Infrastructure, Chinese Scientific Journals database, WANFANG, and Chinese Biomedical Literature database from inception to November 11, 2020. Randomized controlled trials (RCTs) of AZI versus placebo or one positive control drug, AZI plus anti-asthma drugs (AADs) versus the same AADs, and AZI plus AADs versus placebo or one positive control drug plus the same AADs were included. Primary outcomes were number of exacerbations (NoE); score of clinical tools to assess asthma control after treatment; number of days to relieve symptoms with $\beta 2$ agonist (DBA); post-treatment lung function indicators, including FEV1\% of predicted value (pFEV1\%), FVC\% of predicted value (pFVC\%), FEV1/FVC\% of predicted value (pFEV1/FVC\%), and $\mathrm{PEF} \%$ of predicted value (pPEF\%). Secondary outcomes were post-treatment fractional exhaled nitric oxide (FENO); posttreatment eosinophil counts in sputum (sEOS) or blood (bEOS); author self-reported outcomes related to asthma (AROs); and adverse events (AEs). Results: 61 eligible reports from 59 studies were finally included. AZI plus AADs shows no statistically significant difference in $\mathrm{NoE}(\mathrm{RR}=0.49 ; 95 \% \mathrm{CI}, 0.07-3.26 ; \mathrm{P}=0.05)$ and $\mathrm{sEOS}(\mathrm{MD}=-1.13 \% ; 95 \% \mathrm{CI},-3.54 \%-1.29 \%$; $\mathrm{P}=0.36)$ compared to AADs alone. The post-treatment C-ACT score was improved after AZI plus salmeterol and fluticasone $(\mathrm{SF})$ treatment compared to $\mathrm{SF}$ alone $(\mathrm{MD}=2.97 ; 95 \% \mathrm{CI}, 2.39-3.54 ; \mathrm{P}<0.001)$. Results from three studies which could not be meta-analyzed showed that AZI may reduce DBA compared to placebo. AZI combined with AADs could improve posttreatment pFEV1\% (AZI + glucocorticoid $(\mathrm{GC})$ vs GC: MD =6.92\%; 95\% CI, 1.47\%-12.37\%; P = 0.01. AZI + leukotriene receptor antagonist (LTRA) vs LTRA: $\mathrm{MD}=24.88 \% ; 95 \% \mathrm{CI}, 21.47 \%-28.29 \%$; $<0.001$. AZI $+\mathrm{GC}+\mathrm{BA}$ vs GC $+\mathrm{BA}$ : $\mathrm{MD}=12.40 \% ; 95 \% \mathrm{CI}, 9.72 \%-15.08 \% ; \mathrm{P}<0.001)$, pFEV1/FVC\% (AZI + GC vs GC: $\mathrm{MD}=10.24 \% ; 95 \%$ CI, $6.44 \%-$ 14.03\%; P $<0.001$. AZI + GC + BA vs GC + BA: $\mathrm{MD}=9.05 \% ; 95 \%$ CI, $5.66 \%-12.44 \% ; \mathrm{P}<0.001$. AZI + BA vs LTRA $+\mathrm{BA}: \mathrm{MD}=14.48 \% ; 95 \% \mathrm{CI}, 11.84 \%-17.12 \% ; \mathrm{P}<0.001)$, and $\mathrm{pPEF} \%(\mathrm{MD}=7.00 \%, 95 \% \mathrm{CI}, 2.53 \%-11.47 \% ; \mathrm{P}=$ 0.002), but not improve pFVC\% ( $\mathrm{MD}=-10.37 ; 95 \% \mathrm{CI},-20.86 \%-0.12 \% ; \mathrm{P}=0.05)$, compared to AADs alone. Post-treatment bEOS was significantly higher in the AZI group than in the traditional Chinese medicine compound granules group (MD = $0.07 \times 109 / \mathrm{L} ; 95 \% \mathrm{CI}, 0.05 \times 109-0.09 \times 109 ; \mathrm{P}<0.001)$. No statistically significant difference in bEOS after treatment with AZI plus montelukast $(\mathrm{MON})$ and loratadine (LOR) compared to MON and LOR $(\mathrm{MD}=0.03 \times 109 / \mathrm{L} ; 95 \% \mathrm{CI},-0.06 \times 109-$ $0.12 \times 109 ; \mathrm{P}=0.50)$. Meanwhile, AZI combined with AADs did not increase AEs $(\mathrm{RR}=0.76 ; 95 \% \mathrm{CI}, 0.51-1.13 ; \mathrm{P}=0.17)$. Conclusions: AZI was beneficial in improving some clinical symptoms and lung functions in childhood asthma. AZI did not increase AEs when combined with AADs.
\end{abstract}

The efficacy and safety of azithromycin in treatment for childhood asthma: a systematic review and metaanalysis

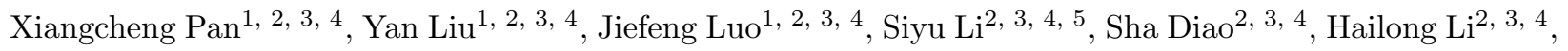


Liang Huang $2,3,4$, Zhijun Jia ${ }^{2,3,4,6}$, Lina Chen ${ }^{4}$, Hanmin $\mathrm{Liu}^{4}{ }^{*}$, Lingli Zhang 1, 2, 3, 4, 5 *

1. West China School of Pharmacy, Sichuan University, Chengdu 610041, China.

2. Department of Pharmacy, West China Second University Hospital, Sichuan University, Chengdu 610041, China.

3. Evidence-Based Pharmacy Center, West China Second University Hospital, Sichuan University, Chengdu 610041, China.

4. Key Laboratory of Birth Defects and Related Diseases of Women and Children (Sichuan University), Ministry of Education, Chengdu 610041, China.

5. West China School of Medicine, Sichuan University, Chengdu 610041, China.

6. Department of Biopharmaceutics, West China School of Pharmacy, Sichuan University, Chengdu 610041, China

* To whom correspondence should be addressed at:

Dr. Lingli Zhang

Evidence-Based Pharmacy Center, West China Second University Hospital, Sichuan University, Chengdu 610041, China.

Tel: 028-85503205; E-mail: zhanglingli@scu.edu.cn

Dr. Hanmin Liu

Key Laboratory of Birth Defects and Related Diseases of Women and Children (Sichuan University), Ministry of Education, Chengdu 610041, China.

E-mail: liuhm@scu.edu.cn

Word Count (Abstract): 542

Word Count (Text): 3247

Number of Tables and Figures: 6

Key Message

Azithromycin has been widely used to treat childhood asthma because of its antibacterial and antiinflammatory effects, but there are inconsistent conclusions on children. The existing systematic reviews included insufficient studies.

The purpose of this study was to further evaluate the efficacy and safety of azithromycin in childhood asthma.

Our findings suggested that azithromycin was beneficial in improving clinical symptoms and some lung function in children, including post-treatment Childhood Asthma Control Test scores, FEV $1 \%$ of predicted value, $\mathrm{FEV}_{1} / \mathrm{FVC} \%$ of predicted value, $\mathrm{PEF} \%$ of predicted value, and did not increase adverse events when combined with other anti-asthma drugs.

\section{Hosted file}

Main Text File.docx available at https://authorea.com/users/416568/articles/524061-theefficacy-and-safety-of-azithromycin-in-treatment-for-childhood-asthma-a-systematicreview-and-meta-analysis

\section{Hosted file}


Figures.docx available at https://authorea.com/users/416568/articles/524061-the-efficacyand-safety-of-azithromycin-in-treatment-for-childhood-asthma-a-systematic-review-andmeta-analysis 